\title{
First-light images from low-dispersion spectrograph-cum-imager on 3.6 m Devasthal Optical Telescope
}

\author{
Amitesh Omar*, T. S. Kumar, B. Krishna Reddy, Jayshreekar Pant and Manoj Mahto
}

A low-dispersion spectrograph-cum-imager has been developed and assembled in ARIES, Nainital. The optical design of the spectrograph consists of a collimator and a focal reducer converting the f/9 beam from the $3.6 \mathrm{~m}$ Devasthal Optical Telescope (DOT) to a nearly f/4.3 beam. The instrument is capable of carrying out broad-band imaging, narrow-band imaging and low-resolution $(\lambda / \Delta \lambda<2000)$ slit spectroscopy in the wavelength range 350-1050 $\mathrm{nm}$. A closed-cycle cryogenically cooled charge-coupled device camera, also assembled in ARIES, is used as the main imaging device for the spectrograph. The first images from the spectrograph on the telescope assert seeinglimited performance free from any significant optical aberration. An i-band image of the galaxy cluster Abell 370 made using the spectrograph shows faint sources down to 25 mag. The quality and sensitivity of the optical spectra of the celestial sources obtained from the spectrograph are according to the expectations from a $3.6 \mathrm{~m}$ telescope. Several new modes of observations such as polarimetry, fast imaging and monitoring of the atmospheric parameters are being included in the spectrograph. Using a test set-up, single optical pulses from the Crab pulsar were detected using the telescope. The spectrograph is one of the main back-end instruments on the $3.6 \mathrm{~m} \mathrm{DOT} \mathrm{for}$ high-sensitivity observations of celestial objects.

Keywords: Astronomical instrumentation, charge-coupled device camera, optical telescope, spectrograph.

A low-dispersion spectrograph-cum-imager (hereafter spectrograph), following the design concepts of various Faint Object Spectrograph and Camera (FOSC)-type instruments ${ }^{1-4}$, has been developed for the $3.6 \mathrm{~m}$ Devasthal Optical Telescope (DOT). The spectrograph, designed and developed with mostly in-country research and development efforts, was assembled in ARIES, Nainital. The FOSC-type instruments are proven to be highly versatile and scientifically productive for low-medium dispersion spectroscopy and imaging of faint celestial sources. The spectrograph consists of a fixed collimator followed by a fixed focal-reducer unit with provisions for inserting optical filters and dispersive elements such as grism, prism and gratings in the optical path. The filters, slits, grisms, etc. are mounted on different motorized rotating wheels for automated and fast operation using a remote computer. If the slit and grism are not inserted in the optical path, the instrument works in non-dispersing imaging mode with focal reduction. In this way, operation mode of the instrument can be changed to imaging or

Amitesh Omar, T. S. Kumar, B. Krishna Reddy, Jayshreekar Pant and Manoj Mahto are in the Aryabhatta Research Institute of Observational Sciences, Manora Peak, Nainital 263 001, India.

*For correspondence. (e-mail: aomar@aries.res.in) spectroscopy within a few seconds by inserting or removing appropriate optical elements in the path. The instrument can also be made to work in the polarization mode if polarizing elements are inserted in the optical path.

The $3.6 \mathrm{~m}$ DOT, a moderate but the largest aperture optical telescope in India, is situated at Devasthal (Nainital) at the geographical location of $29.36^{\circ} \mathrm{N}, 79.69^{\circ} \mathrm{E}$, and $2426 \mathrm{~m}$ above mean sea level in the Himalayan region of Uttarakhand. It uses an f/9 Ritchey-Chrétien (RC) catoptrics system providing the Cassegrain image plane. The telescope points and tracks a celestial source using the altitude over azimuth mount. The primary mirror supported on 69 axial actuators uses the active optics technology, which was originally developed by the European Southern Observatory in 1980s for the new technology telescope ${ }^{5}$. Technical details of DOT and the Devasthal site are provided elsewhere ${ }^{6-8}$. DOT has an internal autoguider to improve tracking accuracy and a wave-front sensor to align the telescope optics. It is suitable to carry out astronomical observations in many areas of galactic and extra-galactic astronomy for observations of star clusters, young stars, supernova and gamma-ray bursts, variability of stars, galaxies, high redshift sources, and active galactic nuclei ${ }^{9}$. Most of these studies require imaging capabilities over several arc-minutes of field of 
view (FoV), and spectroscopic capabilities over the visible waveband with spectral resolving power $(\lambda / \Delta \lambda)$ in the range 100-2000. This spectral resolving power is suitable for detecting majority of spectral lines from faint celestial objects. The spectrographs with higher spectral resolving power, although limited to observations of relatively brighter objects, are also required to fulfil several other science aims envisaged for $\mathrm{DOT}^{9}$.

It was decided during the early phases of the DOT project that a low-dispersion optical spectrograph could be built using the in-country expertise. Subsequently, the design was started in ARIES with help from experts at the Space Applications Centre (SAC) of the Indian Space Research Organisation (ISRO) at Ahmedabad. After several rounds of design optimization in collaboration with the international experts and optical industries, final designs were made and manufacturing of the optics and mechanical components was started. The main optics was fabricated, aligned and tested in the premises of Winlight Systems, France. The precision mechanical components were fabricated and tested in the premises of Pawan Udyog and other small-scale industries in GhaziabadDelhi area as well as using the modern facilities at ARIES. The optics for the calibration unit and wedgeprisms was fabricated in the premises of Hindustan Opticals, Dehradun. The standard grisms and filters were imported from reputed vendors such as Newport Richardson Gratings, USA and Asahi Spectra, Japan. The precision air-slits were manufactured using the laser processing facility at the International Advanced Research Centre for Powder Metallurgy and New Materials (ARCI), Hyderabad. The spectrograph was fully assembled and tested at ARIES. This is the first FOSC-type large-sized astronomy instrument designed, developed and assembled in India. To record images from the spectrograph, a $4096 \times 4096$ pixel charge-coupled device (CCD) camera with an image area of $61.4 \times 61.4 \mathrm{~mm}^{2}$ was also designed, developed and assembled at ARIES in technical collaboration with Herzberg Institute of Astrophysics (HIA), Canada. This article presents a technical summary and the first-light images obtained from the spectrograph. Future upgradation plans are also briefly presented. A comprehensive characterization report and technical details will be published elsewhere.

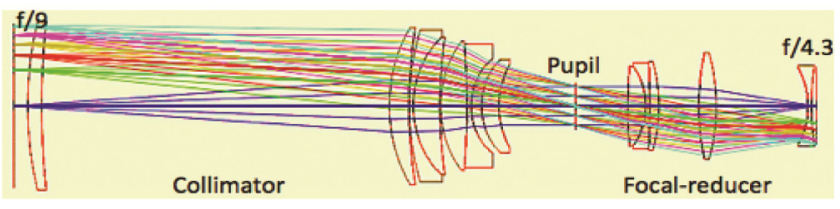

Figure 1. Ray-diagram of the spectrograph optics. The input is $f / 9$ focal plane from the $3.6 \mathrm{~m}$ Devasthal Optical Telescope (DOT) and the output is $\sim f / 4.3$ focal plane imaged on the CCD camera. Total optical track length between the two focal planes is $\sim 1150 \mathrm{~mm}$ and the largest diameter of the lens used is $\sim 200 \mathrm{~mm}$.

\section{Main components and features of the spectrograph}

The spectrograph, a dioptric instrument, consists of a collimator, a focal reducer and four motorized wheels accommodating the inter-changing optical elements, viz. slits, grism/prism/grating, broad-band filters and narrowband filters. A spectral calibration unit is also part of the spectrograph. The optical design of the spectrograph is optimized to obtain $80 \%$ encircled energy within 0.4 arcsec diameter anywhere at the image plane within the central $\sim 10$ arcmin diameter. The length of the optical track from the telescope focal plane to the CCD sensor is $\sim 1150 \mathrm{~mm}$. A preliminary design concept of the spectrograph is presented elsewhere ${ }^{10}$. Figure 1 shows the ray diagram of the optics. The final optical design uses several optical elements made of calcium fluoride $\left(\mathrm{CaF}_{2}\right)$ and $i$-line high-homogeneity glasses S-FSL5, PBM18Y, PBM2Y, PBL1Y and BAL15Y. The $i$-line glasses have low birefringence and provide high internal transmission ( $\sim 98 \%$ for $10 \mathrm{~mm}$ thickness) down to $365 \mathrm{~nm}$ wavelength. The spectrograph converts the $f / 9$ optical beam from the $3.6 \mathrm{~m}$ DOT to a faster beam of $\sim f / 4.3$. The collimator unit consists of seven lenses in five groups with three singlets and two doublets. The focal reducer unit consists of five lenses in three groups with one triplet and two singlets. The last element, a field flattener made of fused silica, is mounted as the window of the CCD vacuum dewar. The field lens in the collimator unit is the largest optical component with a diameter of $\sim 200 \mathrm{~mm}$. The diameter of the pupil plane is $\sim 45 \mathrm{~mm}$. The optical surfaces are polished to a surface accuracy of $\sim 5 \mathrm{~nm}$ rms to minimize light scattering. All the air-glass contact surfaces are also coated with a broad-band anti-reflective (AR) coating to minimize the light losses from the optical surfaces. The AR coating has reflectivity below 1.5\% between 350 and $900 \mathrm{~nm}$.

The broad-band colour-filters are mounted in the collimated beam before the pupil plane providing a maximum usable FoV of $13.6 \times 13.6 \mathrm{arcmin}^{2}$ on the $61.4 \times$ $61.4 \mathrm{~mm}^{2}$ CCD sensor. Presently, the Sloan Digital Sky Survey (SDSS) standard UGRIZ filter system is mounted in five slots. The classical UBVRI filter system will also be made available in near future. There are a total of ten slots in the broad-band filter wheel, which can accommodate five $80 \mathrm{~mm}$ and five $90 \mathrm{~mm}$ diameter filters. The grisms are mounted at the pupil plane. The grisms of $50 \mathrm{~mm} \times 50 \mathrm{~mm}$ size have transmission gratings with $300-600$ lines $/ \mathrm{mm}$. The spectral dispersion is in the range $0.1-0.23 \mathrm{~nm}$ per $15 \mu \mathrm{m}$ pixel $(\sim 0.2 \operatorname{arcsec}$ in sky), or the effective spectral resolving power at 1 arcsec of atmospheric seeing is nearly in the range 500-1000. At the atmospheric seeing near 0.5 arcsec, a resolving power of 2000 may be obtained. An $11^{\circ}$ wedge prism is also available to get very low dispersion $(\sim 6 \mathrm{~nm}$ per $15 \mu \mathrm{m})$ spectrum suitable for slit-less spectroscopy. We are in the 
process of procuring additional gratings at lower resolution and virtual phase holographic grisms to further improve the sensitivities. The narrow-band filters are mounted in the $f / 9$ beam before the telescope focal plane providing a clear FoV of $\sim 11$ arcmin diameter. The narrow-band filter wheel can accommodate five $120 \mathrm{~mm}$ filters. This wheel also has a clear slot of $\sim 192 \mathrm{~mm}$ diameter to enable the light cone for $13.6 \times 13.6 \mathrm{arcmin}^{2}$ FoV to pass through it unobstructed for broad-band imaging. The slits are mounted at the $f / 9$ focal plane of the telescope. The slits provide $\sim 8$ arcmin long FoV with widths ranging between 0.4 and 2 arcsec. The slits were cut on $100 \mu \mathrm{m}$ thick SS-304 and $20 \mu \mathrm{m}$ thick brass foils using a precision laser cutting machine at ARCI, Hyderabad. The electronics control hardware for rotating each of the four wheels using anti-backlash drive mechanism was designed and developed at ARIES with capabilities for remote operation. The wheels and the calibration lamps were operated and controlled using a graphical user interface.

The spectrograph works in imaging and spectroscopy modes. When the photometric imaging observations are desired, only filters are inserted in the optical path, whereas when the spectroscopic observations are desired, grisms and slits are inserted in the optical path. As the bandwidth $(\sim 700 \mathrm{~nm})$ of the usable spectrum (350$1050 \mathrm{~nm}$ ) covered by the spectrograph is more than the lowest wavelength $(\sim 350 \mathrm{~nm})$ of interest, second-order spectrum in the blue region $(350-700 \mathrm{~nm})$ gets superimposed on the first order spectrum in the red region (700$1050 \mathrm{~nm})$. The lower cut-off at $\sim 350 \mathrm{~nm}$ is due to Earth's atmosphere, which does not pass significant light below it and the upper cut-off at $\sim 1050 \mathrm{~nm}$ is due to sensitivity cut-off of the silicon-based CCD sensor. Two filters BG-39 (passing 350-700 nm) and RG-610 (passing $>600 \mathrm{~nm}$ ) - are used as the spectral-order blocking filters to block second- or first-order spectrum from the grating. Either of these filters can be used to obtain pure spectralorder dispersion either in the blue or the red region, without any contamination from the other order.

An in-built calibration unit enables spectral calibration of the dispersion axis on the CCD detector and also to carry out flat-fielding of the spectrum. $\mathrm{Hg}-\mathrm{Ar}$ and $\mathrm{Ne}$ lamps are used for spectral calibration, while a tungstenhalogen lamp emitting continuum light is used for the flat-fielding. A light-integrating sphere is used to get a near-uniform source from the light coming from the lamps. The calibration unit has a biconvex lens, which makes the light from the integrating sphere to converge with a focal ratio of $\sim f / 9$, same as that of the telescope. The converging light path from the lens is perpendicular to the telescope light path and hence a $45^{\circ}$ mirror is used to deflect the converging light from the lamps to focus on the slit plane. The lens and the $45^{\circ}$ mirror of the calibration unit are mounted on two separate motorized linear stages to adjust the focus. When a calibration observation is desired, the lamps are switched on and the lens-mirror system is positioned to focus light on the slit plane and no light from the sky is received as the $45^{\circ}$ mirror blocks the sky. When science observations are desired, the lensmirror system is retracted away from the light path of the telescope and the lamps are switched-off.

The optics and other components are mounted inside a precision-machined enclosure made from aluminum alloys 6061 and 6063. The main optics (collimator and focal reducer) is mounted on a $V$-grooved block. The enclosure is black anodized to minimize light scattering. The weight of the spectrograph is nearly $500 \mathrm{~kg}$. Since the spectrograph is mounted directly on the telescope at one end with the other end free to bend due to gravity, provisions are made to arrest mechanical flexure by providing strengths to the structure using beams and heavyduty turn-buckle fixtures. Since balancing of the altitudeaxis tube of the telescope requires a fixed torque, an additional dummy weight of nearly $1300 \mathrm{~kg}$ is attached along with the spectrograph. This additional weight, attached to the telescope-mounting flange, is structurally detached from the spectrograph and has provisions for adding or removing weight plates to fulfil the balancing requirements. In order to mount and dismount the spectrograph and the dummy weight structure on DOT, a special integration trolley was designed at ARIES and fabricated at small-scale industries in Ghaziabad-Delhi area. Figures 2 and 3 show the internal view of the instrument assembly and a photograph of the instrument as mounted on the $3.6 \mathrm{~m}$ DOT respectively.

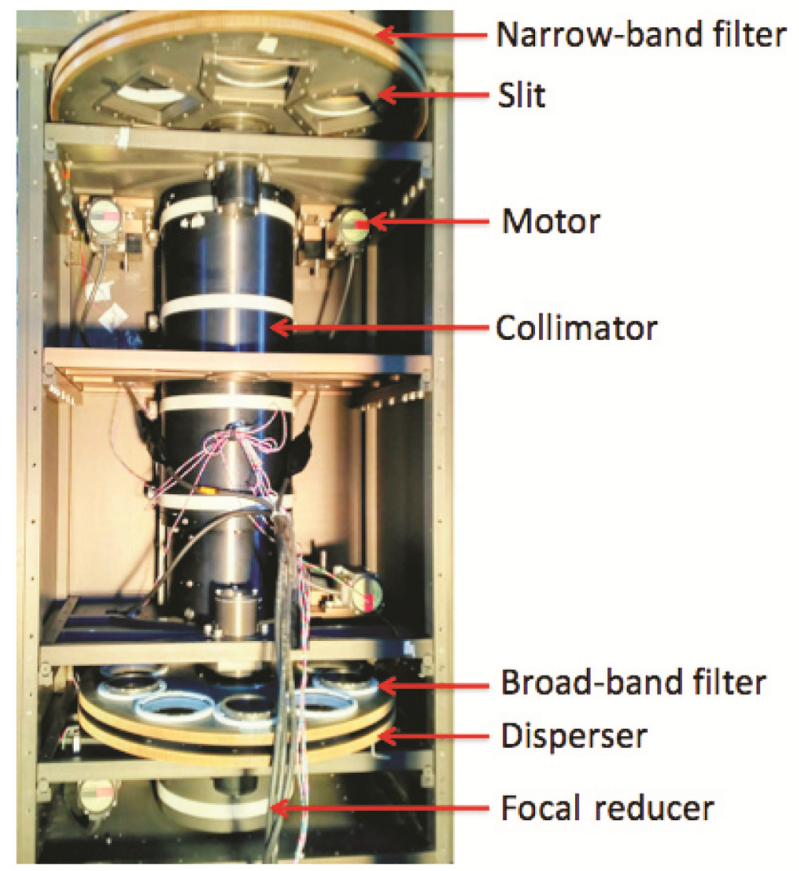

Figure 2. An internal view of the assembled spectrograph showing major components. The light from the telescope enters from the narrowband filter side and the image is formed behind the focal reducer. 
A CCD camera was also designed and assembled in ARIES for the spectrograph. The camera was developed in collaboration with HIA, Canada. It uses a $4096 \times 4096$ format grade-0 back-illuminated E2V 231-84 CCD sensor having square pixels of size $15 \mu \mathrm{m}$. The CCD sensor is a deep-depletion silicon device with multi-layer broad-band coating optimized for fringe-free imaging in the red side. The quantum efficiencies of the CCD are $\sim 84 \%$ at $400 \mathrm{~nm}$ and $\sim 60 \%$ at $900 \mathrm{~nm}$ with peak value of $\sim 90 \%$ near $650 \mathrm{~nm}$. The CCD has quad-amplifier architecture and its $\sim 16$ million pixels can be read from any of the four amplifiers, or through four amplifiers each reading $\sim 4$ million pixels from the four quadrants. The read-out frequency is fixed at $\sim 160 \mathrm{kHz}$ to keep the read-out electronic noise below 10 e rms using a controller from Astronomical Research Cameras, Inc. USA. The CCD sensor is cooled to $-120^{\circ} \mathrm{C}$ using a closed-cycle (JouleThompson) cryogenic heat-exchange system supplied by Brooks Automation, USA. The temperature of the sensor is stabilized and held constant within $0.01^{\circ} \mathrm{C}$ using a proportional-integral-derivative controller and a small heater below the sensor mounting plate. The CCD is operated in non-inverted mode. With these settings, the per pixel dark noise generated is extremely low at a value $\sim 3$ electron per hour. The sensor and a circuit protection board are mounted inside a vacuum dewar. A charcoalfilled getter is used to absorb outgassing inside the dewar. The getter gets activated at cryogenic temperatures and

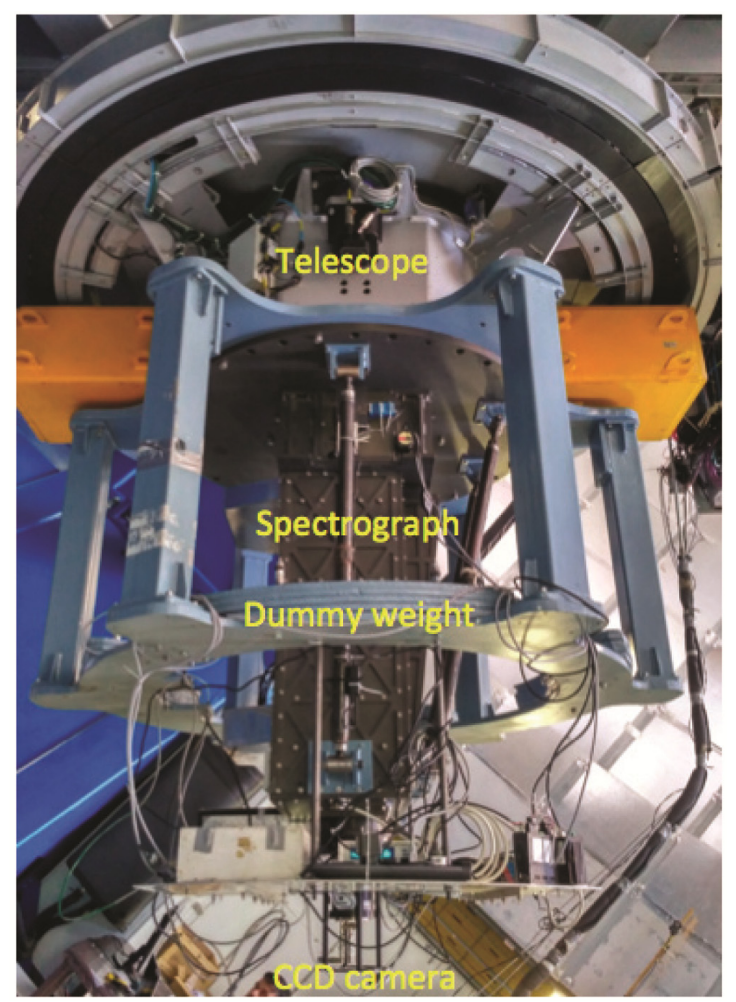

Figure 3. A photograph of the spectrograph mounted on the $3.6 \mathrm{~m}$ DOT. helps in attaining high vacuum inside the dewar. The dewar attains a vacuum $\sim 3 \times 10^{-7}$ torr, when its internal system and the CCD sensor are cooled to $-120^{\circ} \mathrm{C}$. The closed-cycle cooling system with heat-exchanger pipes, although somewhat cumbersome to use on an altitudeazimuth telescope mount, alleviates the need to regularly fill liquid nitrogen commonly used in the CCD cameras for astronomy. The closed-cycle cooling system also makes the CCD dewar compact and less bulky, as the liquid nitrogen holding tank is not needed in such systems. Further details of the CCD system and its characterization will be published elsewhere.

\section{On-sky performance}

The spectrograph has been mounted on the $3.6 \mathrm{~m}$ DOT a few times to analyse the on-sky performance. The instrument was used in both imaging and spectroscopy modes for the observations of stars, ionized star-forming regions and galaxies. Figure 4 presents a calibrated image of the galaxy cluster Abell 370 in the SDSS $i$-band (700$850 \mathrm{~nm}$ ) with total exposure time of 55 min taken in 11 frames of $5 \mathrm{~min}$ each on 14 November 2017. The photon counts in the image were converted to flux (magnitude) using the calibrated image of the same region available from the SDSS archive. The image has full-width at halfmaximum (FWHM) of $\sim 1.5 \operatorname{arcsec}$ and the image frames were taken when the object was in the west near the zenith distance of $\sim 55^{\circ}$. The $i$-band sky brightness at Devasthal at the time of the observations determined from this image is $\sim 20 \mathrm{mag} \operatorname{arcsec}{ }^{2}$, which is at par with several dark observing sites worldwide. The background sky is uniform (flat) in the processed image to a high accuracy of $\sim 0.1 \% \mathrm{rms}$. The faintest sources detected in this image are i 24-25 mag with signal-to-noise ratio between 3 and 5 . The locations of the faintest sources in this image match with those in the deep image of the same region, available from the Hubble Space Telescope (HST) Legacy program. The famous giant gravitational lens arc is also clearly visible in this image. A 25 mag source has a feeble effective photon rate of about 1 photon/sec through the spectrograph on the $3.6 \mathrm{~m}$ DOT. To put this faint detection in perspective, human eyes can detect a star of about $7 \mathrm{mag}$, which has about 10 million times higher photon rate than the 25 mag source detected here. The best-recorded stellar images from the spectrograph have FWHM $\sim 1.5$ arcsec in the $u$-band $(\sim 350 \mathrm{~nm})$ and $\sim 1.1$ arcsec in the $z$-band $(\sim 900 \mathrm{~nm})$. The high fidelity in the DOT images and excellent correspondence in terms of faint detections with the HST images imply that the spectrograph on the $3.6 \mathrm{~m}$ DOT is capable of making very deep images of faint sources to a high accuracy. It is worth mentioning here that as the CCD camera used in this spectrograph is sensitive in the red side, scientific observations of the near-infrared bright sources such as 


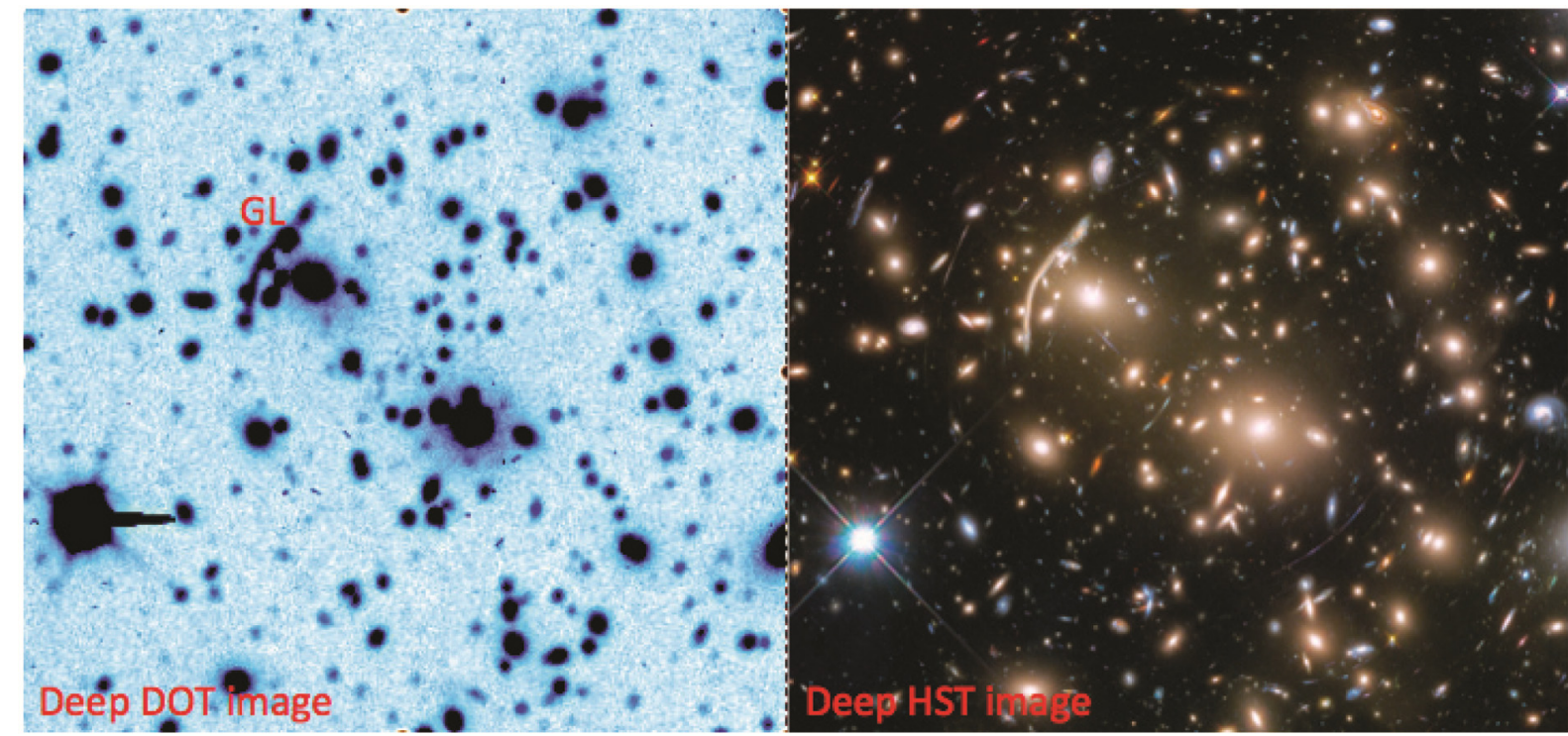

Figure 4. (Left) An $i$-band image of the central region $\left(\sim 3^{\prime} \times 3^{\prime}\right)$ of the galaxy cluster Abell 370 made using observations from the spectrograph on the $3.6 \mathrm{~m}$ DOT in the imaging mode. (Right) A deep image made using the HST (credit: NASA, ESA, J. Lotz and the Hubble Frontier Fields team of STScI) is reproduced to make a comparison of the locations of the faint detected sources. Most of the diffuse objects in the image are galaxies. The detection limit in the DOT image of 55 min exposure is at $\sim 25$ mag with a photometric precision of 0.3 mag. The prominent gravitational lens arc is marked as GL.

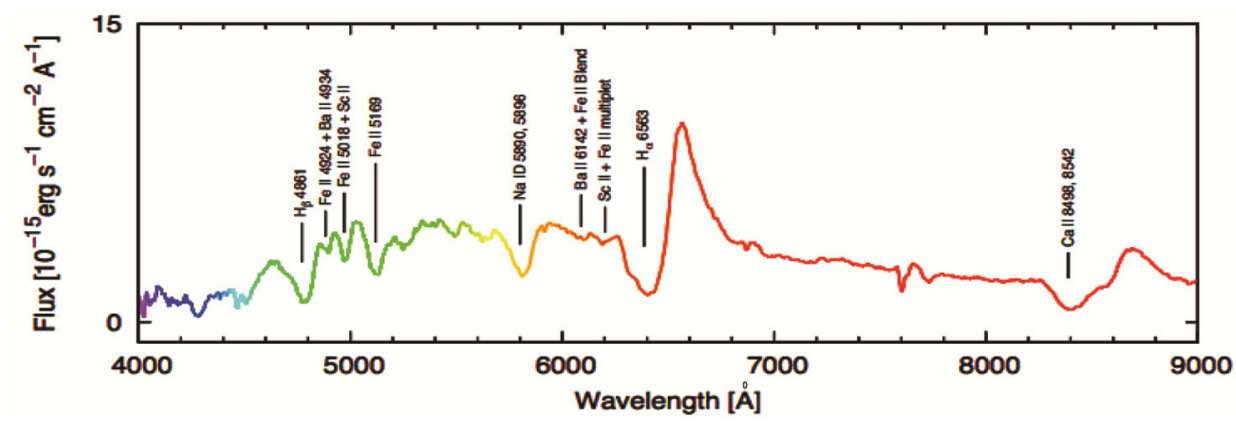

Figure 5. Optical spectrum of the supernova $2017 \mathrm{gmr}$ using the spectrograph on $3.6 \mathrm{~m}$ DOT in a single exposure of $5 \mathrm{~min}$. The prominent elemental lines are marked. The spectrum has average resolution $(\Delta \lambda)$ of $\sim 10 \AA$. The rms noise values $\left(\mathrm{erg} / \mathrm{cm}^{2} / \mathrm{s} / \AA\right)$ are $4 \times 10^{-16}$ near $4500 \AA, 2 \times 10^{-16}$ near $6000 \AA, 0.8 \times 10^{-16}$ near $7500 \AA$ and $1 \times 10^{-16}$ near $9000 \AA$. The peak detections of the $\mathrm{H} \alpha$ and $\mathrm{H} \beta$ lines are made at the signal-to-noise ratio of 70 and 20 respectively.

high-redshift galaxies and extremely cold or low-mass stars (white dwarfs, brown dwarfs, etc.) will also be possible using the spectrograph.

Figure 5 shows the calibrated spectrum of the extragalactic supernova (SN) 2017 gmr using observations from the spectrograph taken on 17 November 2017, when the supernova was only about 73 days old. At the time of the observations, the supernova had visual magnitude of $\sim 15$. The line strengths of the prominent emission lines of the elements $\mathrm{H}, \mathrm{C}, \mathrm{N}, \mathrm{O}, \mathrm{Fe}$ as marked in Figure 5, indicate that this supernova is hydrogen-rich (type-II) and most likely produced from the explosion of a runaway massive star. It is important to mention that this spectrum was generated in a single exposure of 5 min duration, covering the entire wavelength range between 400 and $900 \mathrm{~nm}$. The spectrum has an excellent correspondence in terms of the line features and strengths with the archived spectra of similar types of supernovae. The supernovae at this stage evolve rapidly and the line features change every few days. The spectroscopic observations of supernovae over regular intervals provide crucial information on evolution and nucleo-synthesis of various elements in the Universe. The high fidelity of the optical spectrum indicates that this spectrograph on the $3.6 \mathrm{~m}$ DOT will be valuable for spectroscopic studies of faint celestial objects.

\section{Possible upgradations}

Several new modes of observations are possible to include in the FOSC-type spectrograph presented here. A 

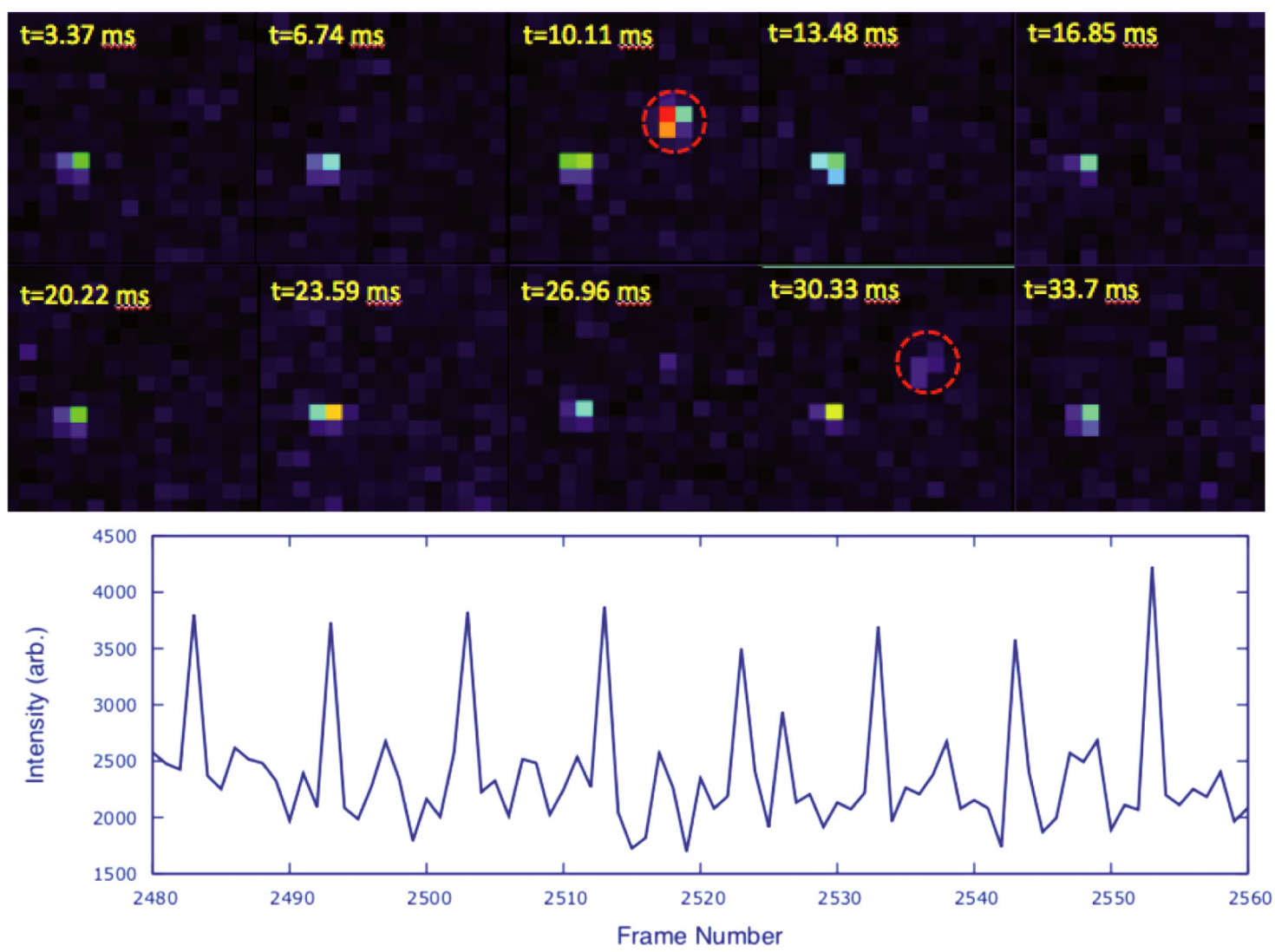

Figure 6. (Top) The millisecond images for one cycle of pulse emission from the Crab pulsar (marked by red circles), recorded using an EM-CCD camera mounted on the $3.6 \mathrm{~m}$ DOT. The object with persistent emission to the left of the pulsar is a star. (Bottom) Intensity profile of optical pulses from the Crab pulsar. The abscissa represents frame numbers with time separation of $\sim 3.37 \mathrm{~ms}$. The pulsar has a known period of $\sim 33.7 \mathrm{~ms}$. Both strong main pulse $(t \sim 10 \mathrm{~ms})$ and weak inter-pulse $(t \sim 27-30 \mathrm{~ms})$ are detected.

polarimetry mode is planned to be included using a Wollaston prism and an appropriate wave-plate. This mode will enable measurement of polarization of the light coming from various celestial objects. It is also possible to include multi-object spectroscopy mode using a slit mask customized for a field. A fast-imaging mode to measure small variations in the flux of variable sources at millisecond cadence is being included in the present design. For this mode, a frame-transfer electron-multiplying (EM)CCD camera is presently being tested on the $3.6 \mathrm{~m}$ DOT. This CCD camera provides a FoV of $1.7 \times 1.7 \mathrm{arcmin}^{2}$ with readout rate up to $\sim 350$ frames per second. A global position system (GPS)-based time-event-monitoring system expected to make time-stamping of the image frames at an accuracy $<0.1 \mathrm{~ms}$ is also presently under testing. A test observation made using the EM-CCD camera directly on the $3.6 \mathrm{~m}$ DOT fetched exciting results on the observations of the Crab pulsar (PSR B0531+21), which is a young neutron star spinning rapidly with a rotation period of $\sim 33.7 \mathrm{~ms}$. Figure 6 shows repeating optical pulses from the Crab pulsar, detected from the $3.6 \mathrm{~m}$ DOT. Each data point here corresponds to $\sim 3.37 \mathrm{~ms}$ of effective cadence (exposure + read-out time). A sensitivity estimate indicates that a $\sim 17$ mag source can be expected to get detected in $10 \mathrm{~ms}$ integration using a high-sensitivity EM-CCD camera. This set-up can also be effectively combined with a low-dispersion prism and an appropriate multi-band-pass filter to carry out multi-colour imaging of some bright ( $<17 \mathrm{mag}$ ) sources at fast cadence, similar to a multi-channel fast photometer.

The annular FoV beyond the central $13.6 \times 13.6$ arc$\min ^{2}$ used by the spectrograph is also available at the $f / 9$ beam as the $3.6 \mathrm{~m}$ DOT has a total usable FoV of $\sim 30$ arcmin diameter at the Cassegrain focal plane. This annular FoV can be imaged by another CCD camera directly in the $f / 9$ beam to provide an independent measurement of the sky parameters, such as extinction. The CCD camera can also be used for external guiding to improve tracking accuracy. A set-up using a $0.5^{\circ}$ wedge prism at the pupil plane is also being tested in the spectrograph to measure atmospheric seeing parameters based on differential image motion monitor (DIMM) principles $^{11}$. This DIMM set-up and continuous extinction measurement set-up within the spectrograph will be valuable for obtaining accurate photometry of the celestial objects. 


\section{Conclusions}

A spectrograph similar to the popular and versatile astronomy instrument FOSC has been designed and developed in ARIES. This is the first large-sized FOSCtype astronomy spectrograph designed and assembled within the country, thanks to the expertise available in various Indian Government institutes and organizations, industries, and technical support received from some organizations abroad, most notably from Belgium, Canada and France. A large-format closed-cycle cryogenically cooled CCD camera was also developed and assembled in ARIES for the spectrograph. The spectrograph and the CCD camera were mounted on the $3.6 \mathrm{~m}$ DOT for conducting various tests. The first-light results showed satisfactory performance of the spectrograph. Images as deep as $\sim 25 \mathrm{mag}$ in the SDSS $i$-band with excellent uniformity across the full FoV have been made. The spectroscopy was successfully performed on faint celestial objects. The individual optical pulses from the Crab pulsar were detected using a fast EM-CCD camera on the telescope. In future, the fast read-out mode coupled with a GPS timing system in the spectrograph will enable precise multi-wavelength coordinated optical follow-up observations of fast transients along with other telescopes such as the upgraded Giant Meterwave Radio Telescope $(\text { GMRT })^{12}$ in radio wavebands and AstroSat ${ }^{13}$ in highenergy bands. A polarimetry mode and other features such as seeing and extinction monitor, and external guiding are also planned to be included in the spectrograph to further enhance its capabilities.

1. Buzzoni, B. et al., The ESO Faint Object Spectrograph and Camera (EFOSC). ESO Messenger, 1984, 38, 9-13.

2. Andersen, J. et al., New power for the Danish 1.54-m telescope. ESO Messenger, 1995, 79, 12-14.

3. Kashikawa, N. et al., FOCAS: the Faint Object Camera and Spectrograph for the Subaru Telescope. Publ. Astron. Soc. Jpn, 2002, 54(6), 819-832.

4. Hook, I. M. et al., The Gemini-North Multi-Object Spectrograph: performance in imaging, long-slit, and multi-object spectroscopic modes. Publ. Astron. Soc. Pacific, 2004, 116(819), 425-440.

5. Hardy, J. W., Active optics: a new technology for the control of light. Proc. IEEE, 1978, 66, 651
6. Ninane, N., Carlo, F. and Kumar, B., The $3.6 \mathrm{~m}$ Indo-Belgium Devasthal Optical Telescope: general description. Proc. SPIE, 2012, 8444, IV.

7. Kumar, B. et al., 3.6-m Devasthal Optical Telescope project: completion and first results. Bull. Soc. R. Sci. Liega, 2018, 87, 29 41.

8. Sagar, R. et al., Evaluation of Devasthal site for optical astronomical observations. Astron. Astrophys. Suppl., 2000, 144, 349-362.

9. Omar, A., Kumar, B., Gopinathan, M. and Sagar, R., Scientific capabilities and advantages of the 3.6 metre optical telescope at Devasthal, Uttarakhand. Curr. Sci., 2017, 113, 682-685.

10. Omar, A., Yadav, R. K. S., Shukla, V., Mondal, S. and Pant, J., Design of FOSC for $360-\mathrm{cm}$ Devasthal optical telescope. Proc. SPIE, 2012, 8446.

11. Sarazin, M. and Roddier, F., The ESO differential image motion monitor. Astron. Astrophys., 1990, 227, 294.

12. Gupta, Y. et al., The upgraded GMRT: opening new windows on the radio Universe. Curr. Sci., 2017, 113, 707-714.

13. Singh, K. P. and Bhattacharya, D., Multi-colour hues of the Universe observed with AstroSat. Curr. Sci., 2017, 113, 602-609.

ACKNOWLEDGEMENTS. The assembly of this instrument could not have been possible without the help received from several organizations, industries, and individual experts. We acknowledge academic, technical and administrative support received from the staff of ARIES, too many to name here individually, to build this instrument and get first light on it through the $3.6 \mathrm{~m}$ DOT. We thank SAC (ISRO), Ahmedabad; ARCI, Hyderabad; the Department of Science and Technology (DST), Government of India and ARIES Governing Council and Project Management Board of the $3.6 \mathrm{~m}$ DOT project for support. We also thank the members of the review committee of the project for providing constructive inputs at various stages; A. Paswan, R. Dastidar and Dr K. Misra for help during the observations and in data reduction; the DOT operation team led by Dr Brijesh Kumar and Dr Wahab Uddin (Director) for their inputs and supports; Profs P. C. Agrawal, R. Sagar, S. N. Tandon, G. Srinivasan, S. Ananthakrishnan, T. P. Prabhu, G. Swarup, S. K. Joshi and P. Asthana for their encouragement to develop the spectrograph within the country; R. K. S. Yadav, S. Mondal, V. Shukla, S. Yadava, N. Nanjappa, T. Bangia, A. Misra, A. N. Ramaprakash, G. Raskin, J. Surdej. T. Hardy and G. Burley for useful discussions; HIA, National Research Council, Canada for help to develop the CCD camera and Profs G. Padmanabhan and R. Bathe for help to develop slits in ARCI, Hyderabad.

Received 31 October 2018; revised accepted 8 February 2018

doi: $10.18520 / \mathrm{cs} / \mathrm{v} 116 / \mathrm{i} 9 / 1472-1478$ 